Disclosure of Interest: None declared

DOI: 10.1136/annrheumdis-2017-eular.3658

\section{FRI0006 PROTEIN CITRULLINATIONS BY PAD ENZYMES PROMOTE DENDRITIC CELL TRANSDIFFERENTIATION INTO OSTEOCLAST AND GENERATE TARGETS FOR RA-SPECIFIC ANTIBODIES}

B. Réthi ${ }^{1}$, A. Krishnamurthy ${ }^{1}$, J. Ytterberg ${ }^{1}$, M. Sun ${ }^{1}$, V. Joshua ${ }^{1}$,

H. Wähämaa ${ }^{1}$, N. Tarasova ${ }^{2}$, J. Steen ${ }^{1}$, V. Malmström ${ }^{1}$, A.I. Catrina ${ }^{1}$

${ }^{1}$ Department of Medicine, Center for Molecular Medicine, Karolinska Institutet \& Karolinska University Hospital; ${ }^{2}$ Department of Medical Biochemistry and Biophysics, Karolinska Institutet, Stockholm, Sweden

Background: Immature dendritic cells (DCs) are able to trans-differentiate into osteoclasts (OCs) although the mechanisms regulating this process are little understood. We have recently described an important role for protein citrulliantion and peptidylarginine deiminase (PAD) enzyme activity in the regulation of OC development (1).

Objectives: We studied the molecular bases of DC-OC trans-differentiation and aimed at understanding the role of protein citrullination in this process.

Methods: Monocyte-derived DCs and peripheral blood CD1C+ DCs were cultured in the presence of osteoclastogenic cytokines. Polyclonal ACPAs were isolated from the serum of RA patients and applied in OC cultures. DC and OC differentiation was analyzed in vitro using gene expression analyses, flow cytometry-based methods, DC-T cell co-culture experiments, tartrate-resistant acid phosphatase stainings and osteolysis assays. Protein citrullination was monitored with the help of mass spectrometry. PAD activity was measured by ELISA.

Results: Different DC types showed different capacities to develop into OCs. OC-prone DCs were characterized by little immunogenicity and their development was potentiated by the increase of lactic acid, a side product of glycolytic metabolism. The more immunogenic DC types, characterized by prominent ability to migrate towards secondary lymphoid tissues and trigger $\mathrm{T}$ cell activation, showed a limited capacity to develop into OCs (2). The differentiation switch towards the $\mathrm{OC}$ lineage was associated with increased activity of the Protein Arginine Deiminase (PAD) enzymes and with higher level of protein citrullination in $\mathrm{DCs}$. The PAD inhibitor Cl-Amidine efficiently interfered with OC development form DC precursors. In addition, the deposition of citrullinated proteins on the cell surface made the cells sensitive for anti-citrullinated protein autoantibodies, which could further stimulate DC-OC trans-differentiation through inducing the cytokine IL-8.

Conclusions: Our results indicated that DCs are heterogenic in their ability to form OCs and lineages for immunostimulatory and OC-prone DCs might separate early during DC differentiation. Plasticity towards OC differentiation might be influenced by the metabolic environment and the upregulation of PAD activity in DCs.

References:

[1] Krishnamurthy A et al. Ann Rheum Dis 2016.

[2] Nasi A et al. J Immunol 2013.

Disclosure of Interest: None declared

DOI: 10.1136/annrheumdis-2017-eular.5971

\section{FRI0007 METHYL GALLATE INHIBITS OSTEOCLAST FORMATION AND FUNCTION THROUGH SUPPRESSING THE AKT AND BTK-PLC $\gamma 2$-CA2+ SIGNALING, AND PREVENTS LPS-INDUCED BONE LOSS}

M.-S. Lee ${ }^{1}$, J.M. Baek ${ }^{2}$, J.-Y. Kim ${ }^{2}$, W.-H. Yoo ${ }^{3}$, S.-J. Hong ${ }^{4}$, C.-H. Lee ${ }^{1}$ ${ }^{1}$ Internal Medicine, Wonkwang University Hospital; ${ }^{2}$ Anatomy, School of Medicine, Wonkwang University, Iksan; ${ }^{3}$ Internal Medicine, Chonbuk National University Medical School, Jeonju; ${ }^{4}$ Internal Medicine, Kyung Hee University School of Medicine, Seoul, Korea, Republic Of

Background: Methyl gallate, a plant-derived phenolic constituent has been known to possess numerous pharmacological features against inflammation, oxidation, and cancer. But so far, there have been no evidences to describe relationship between methyl gallate and bone metabolism.

Objectives: In order to propose a promising candidate for osteoporosis, we performed experiments in this study by using methyl gallate.

Methods: we performed screening of methyl gallate utilizing TRAP staining and revealed intracellular mechanisms responsible for methyl gallate-mediated regulation of osteoclastogenesis through western blotting and quantitative RTPCR. Also, we assessed the role of methyl gallate on characteristics of mature osteoclasts. we used LPS-induced bone loss mice as a model of osteoporosis and analyzed using micro-CT system and the right femurs were stained with TRAP and H\&E.

Results: we observed that methyl gallate significantly suppressed osteoclast formation through Akt and Btk-PLC $\gamma 2-\mathrm{Ca}^{2+}$ signaling. The blockade of these pathways was reconfirmed through transduction of CA-Akt retrovirus and evaluation of $\mathrm{Ca}^{2+}$ influx intensity stained with Fluo-3/AM. Indeed, methyl gallate down-regulated the formation of actin ring-positive osteoclasts and resorption pit areas. In agreement with in vitro results, we found that the administration

of methyl gallate restored osteoporotic phenotype stimulated by acute systemic injection of LPS in vivo through micro-CT and histology.

A
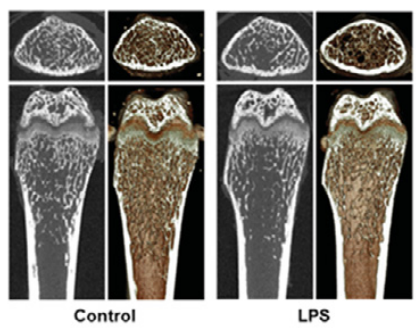

LPS

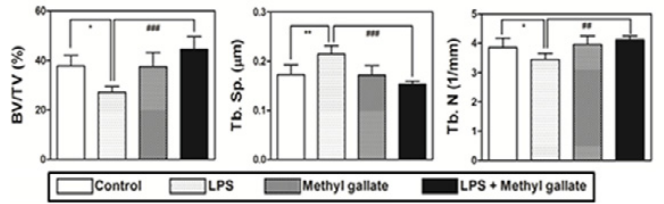

C

Control

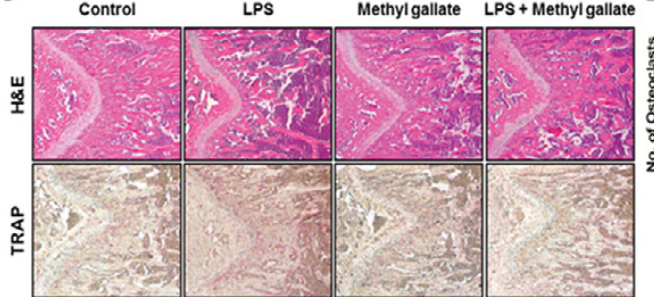

D

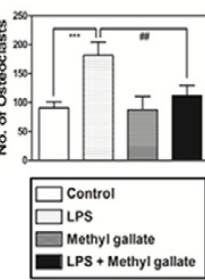

Conclusions: Consequently, the overall data strongly indicated that methyl gallate could be a useful substance for development of plant-based anti-osteoporotic agent.

Disclosure of Interest: None declared

DOI: 10.1136/annrheumdis-2017-eular.2141

\section{FRI0008 EFFECTS OF THE HUMAN IL4-10 FUSION PROTEIN IN THE CANINE GROOVE MODEL OF OSTEOARTHRITIS}

E.M. Van Helvoort ${ }^{1}$, J. Popov-Celeketic ${ }^{1}$, K. Coeleveld ${ }^{1}$, M.A. Tryfonidou ${ }^{2}$, F.P. Lafeber ${ }^{1}$, S.C. Mastbergen ${ }^{1} .{ }^{1}$ Rheumatology and Clinical Immunology, University Medical Centre Utrecht; ${ }^{2}$ Clinical Sciences of Companion Animals, Faculty of Veterinary Medicine, University Utrecht, Utrecht, Netherlands

Background: Ideally a disease modifying osteoarthritis drug (DMOAD) combines treatment for pain, tissue damage and inflammation, all in one molecule. Intraarticular application of a DMOAD brings additional value to treatment for two reasons, (i) lower risk of systemic side effects and (ii) higher drug concentration and potentially improved penetration of non-vascularized articular cartilage. Interleukin-4 (IL-4) and Interleukin-10 (IL-10) have been shown to prevent join degeneration and can work synergistically ${ }^{1}$.

Objectives: This study evaluates the DMOAD activity of repetitive intra-articular injections with a human fusion protein of IL-4 and IL-10 (hIL4-10FP) in the canine Groove model of osteoarthritis (OA).

Methods: In 8 dogs joint degeneration was induced according to the Groove model. Six weeks after surgery dogs were treated with ten weekly intra-articular injections of either hIL4-10FP $(n=4)$ or PBS $(n=4)$. Subsequently, dogs were euthanized and cartilage and synovium were harvested. Cartilage damage and synovial inflammation were macroscopically evaluated. Proteoglycan release and content were determined ex vivo by staining of glycosaminoglycans (GAGs) with Alcian Blue. Proteoglycan synthesis was measured by ${ }^{35} \mathrm{SO}_{4}^{2-}$ incorporation and precipitation with cetylpyridium chloride and liquid scintillation analysis of ${ }^{35} \mathrm{SO}_{4}^{2-}$ labeled GAGs. Potential antibody formation against hIL4-10FP was evaluated with ELISA and a cell based assay. Immunohistochemistry of CD79 $\alpha$ and CD10 was used to evaluate the presence of B-cells in synovium.

Results: Affected knees of PBS treated dogs showed enhanced macroscopic cartilage damage compared to their controls ( 0.31 vs $2.44, p=0.068$ ). Also differences in proteoglycan release, content and synthesis indicated a degenerative state in the affected knees.

Unexpectedly, enhanced synovial inflammation was observed in hIL4-10FP treated joints compared to PBS treated joints, demonstrated by enhanced macroscopic (3.5 vs 2.3 out of 5) and histologic (2.5 vs 1.8 out of 6 ) scores. CD79 $\alpha$ and CD10 showed enhanced expression in synovium of the hIL4-10FP group compared to the PBS group, although not statistically significant (fig 1). Additional analyzes showed that hIL4-10FP was immunogenic in dogs after multiple injections. Formation of neutralizing antibodies was shown in a cell based assay where the activity of hIL4-10FP was inhibited in the presence of serum of hIL4-10FP treated dogs (fig 2). Despite the enhanced inflammatory response 